\section{Automedicação em menores de cinco anos em municípios do Pará e Piauí: prevalência e fatores associados}

\section{Self-medication of children aged under five years in municipalities in the States of Pará and Piauí: prevalence and associated factors}

Ivana da Cruz Goulart 1

Juraci Almeida Cesar 2

David Alejandro Gonzalez-Chica 3

Nelson Arns Neumann 4

1,2 Programa de Pós-Graduação em Ciências da Saúde. Faculdade de Medicina. Universidade Federal do Rio Grande (FURG). Rua General Osório, s/n. $4^{\circ}$ andar. Centro. Campus Saúde - Área Acadêmica Prof. Newton Azevedo. Rio Grande, RS, Brasil. CEP: 96.201-900. E-mail: juraci.cesar@gmail.com

${ }^{3}$ Departamento de Nutrição. Universidade Federal de Santa Catarina. Florianópolis, SC, Brasil.

${ }^{4}$ Coordenação Nacional da Pastoral da Criança. Curitiba, PR, Brasil.

\section{Abstract}

Objectives: to measure the prevalence of and identify factors associated with the self-medication of children aged under five years in the municipalities of Caracol in the State of Piaui, and Garrafão do Norte in the State of Pará.

Methods: a cross-sectional method, systematic sampling and a standard household visit questionnaire were used to investigate various characteristics of the mothers, families and children. The chi-squared test was used to compare proportions and Poisson's regression adjusted for robustness of variance for multivariate analysis.

Results: of the 590 children studied in Caracol and 1081 in Garrafão, 30\% and 25\% respectively had been self-medicated in the preceding 15 days. After adjustment for various confounding variables, the fact of not having succeeded in obtaining care for a sick child in the previous 15 days in both municipalities, and that of residing more than $1 \mathrm{~km}$ from health services in Caracol and of the mother having done paid work in the previous 12 months, in Garrafão, proved to be significantly associated with selfmedication among children aged under five years.

Conclusions: the prevalence of self-medication by mothers of children aged under five years in the municipalities under study, although lower than that observed in other parts of Brazil, resulted above all from not being able to gain access to medical assistance.

Key words Self-medication, Drugs, Child health, Child

\section{Resumo}

Objetivos: medir a prevalência e identificar fatores associados à automedicação em crianças menores de cinco anos nos municipios de Caracol no Estado do Piauí, e Garrafão do Norte no Pará.

Métodos: utilizando-se de delineamento transversal, amostragem sistemática e utilização de questionário padrão por meio de visita domiciliar, foram investigadas diversas características das mães, das famílias e das crianças. Utilizou-se teste do quiquadrado para comparar proporções e regressão de Poisson com ajuste robusto da variância na análise multivariável.

Resultados: dentre as 590 crianças estudadas em Caracol e 1081 em Garrafão, 30\% e 25\% haviam sido automedicadas nos últimos 15 dias, respectivamente. Após análise ajustada para diversas variáveis confundidoras, o fato de não conseguir atendimento para o filho doente nos últimos 15 dias nos dois municipios, assim como de residir há mais de $1 \mathrm{~km}$ dos serviços de saúde em Caracol e de a mãe exercer trabalho remunerado nos últimos 12 meses em Garrafão mostraram-se significativamente associadas à automedicação entre menores de cinco anos.

Conclusões: a prevalência de automedicação por parte das mães para menores de cinco anos nos municípios estudados, apesar de inferior à observada em outras localidades brasileiras, decorreu, sobretudo, da dificuldade de realização de consulta médica.

Palavras-chave Automedicação, Medicamentos, Saúde infantil, Criança 


\section{Introdução}

A automedicação é um dos problemas de maior complexidade em saúde pública. A Organização Mundial da Saúde (OMS) estima que pelo menos metade dos medicamentos seja prescrita ou vendida de forma inadequada e, destes, cerca de $50 \%$ são consumidos de forma imprópria. ${ }^{1}$ Isto é resultado do livre comércio para muitos deles, do uso abusivo de medicamento por conta própria, da falta de fiscalização, do incentivo à medicalização por parte dos fabricantes e do impulso do ser humano em consumir medicamentos. ${ }^{2,3}$

O Sistema Nacional de Informações TóxicoFarmacológicas (SINITOX), 4 órgão vinculado ao Ministério da Saúde, que tem por função coletar, compilar, analisar e divulgar dados referentes à intoxicação e envenenamento mostra que entre 2000 e 2008 a taxa de intoxicação por medicamento passou de $15 \%$ para $31 \%$, e que um terço destas intoxicações ocorriam entre menores de cinco anos, com taxa de letalidade de $7 \% .5$

A principal causa de intoxicação medicamentosa entre menores de cinco anos advém da automedicação, que se refere à utilização de medicamentos sem a devida prescrição, orientação e ou acompanhamento médico. 6 Além da intoxicação, a automedicação pode levar a dependência medicamentosa, mascaramento de doenças, enfermidades iatrogênicas, entre outras consequências danosas.6-8 A automedicação é ainda favorecida pela indisponibilidade de atendimento médico e pela falta de medicamentos básicos nos serviços de saúde. 9

A automedicação na infância é um fenômeno comum. Em Pelotas, RS, 56\% das mães cujos filhos pertenciam à coorte de 1982 recorreram à automedicação nos 15 dias antecedentes à entrevista. ${ }^{2}$ Dados da coorte de 2004 revelam que o fenômeno da automedicação vem aumentando, passando de 11\% aos três meses, para $26 \%$ aos 12 meses e $34 \%$ aos 24 meses. ${ }^{9}$ Em Limeira e Piracicaba, SP, alcançou 57\% entre crianças e adolescentes em 200710 em Teresópolis, RJ, foi de 50\% em 2004,11 e em Tubarão, SC, de 74\% em 2005.12

Estudos sobre automedicação entre crianças são pouco frequentes e restritos a centros urbanos e voltados à descrição de prevalências. A realização de um amplo estudo com menores de cinco anos nos municípios de Caracol, Estado do Piauí (PI), e Garrafão do Norte no Estado do Pará (PA), permitiu medir a prevalência e identificar fatores associados à automedicação nesta população.

\section{Métodos}

Este estudo foi conduzido nos municípios de Caracol (PI) e Garrafão do Norte (PA). De acordo com o Instituto Brasileiro de Geografia e Estatística (IBGE), 13 Caracol, localizado no sul do Estado do Piauí, Região Nordeste, possuía 10.838 habitantes e produto interno bruto (PIB) per capita/ano de R $\$$ 2.230,00. Garrafão do Norte, localizado na Região Norte, no Estado do Pará, próximo à divisa com o Estado do Maranhão, possuía 25.538 habitantes e PIB per capita/ano de R $\$ 2.480,00.13$ A taxa de mortalidade infantil nestes dois municípios em 2005 quando estes dados foram coletados havia sido estimada pelo IBGE em 29/1000 para Caracol e em 41/1000 para Garrafão. 13

A população alvo deste estudo foi composta por crianças com idade entre 0 e 59 meses residentes nestas localidades entre os meses de julho e setembro de 2005. Crianças que estavam hospitalizadas neste período foram excluídas do estudo. O delineamento utilizado foi do tipo transversal (seccional ou de prevalência).

Considerando que o objetivo deste estudo era avaliar diversos indicadores relacionados à saúde infantil, particularmente aqueles relacionados ao padrão de morbidade, de utilização de serviços de saúde e estado nutricional, o cálculo do tamanho da amostra, tendo automedicação entre menores de cinco anos como desfecho, teve de ser feito a posteriori. Para uma prevalência encontrada de utilização de medicamentos de $27 \%$ entre menores de cinco anos nestas duas localidades, nível de confiança de $95 \%$ e margem de erro de 2,1 pontos percentuais, o presente estudo deveria incluir pelo menos 1646 crianças. Em relação ao tamanho mínimo de amostra para o estudo da associação foram utilizados os seguintes parâmetros: erro alfa de 0,05 , erro beta de 0,20 , razão de prevalências de 1,5 e frequência de exposição variando entre $10 \%$ e $90 \%$ nos expostos e entre $15 \%$ e $25 \%$ nos não expostos, o estudo deveria incluir 1457 nos dois municípios. Este valor já se encontra acrescido de $15 \%$ para controle de potenciais fatores de confusão e $10 \%$ para perdas. 14

Para alcançar o número desejado de crianças no município de Caracol, foi necessário visitar metade dos seus domicílios. Em Garrafão, município 2,6 vezes mais populoso que Caracol, decidiu-se visitar apenas um terço dos domicílios. Para a escolha dos domicílios realizou-se mapeamento das áreas e numeração de diversos pontos em cada uma delas que, em geral, era uma esquina na área urbana ou cruzamento de estradas em área rural. Em seguida, realizava-se sorteio aleatório de cada um destes 
pontos, a partir do qual todos os domicílios eram visitados sempre em sentido horário. Assim, em Caracol, visitava-se um domicilio e pulava-se outro, enquanto em Garrafão do Norte, escolhia-se um domicilio e os dois subsequentes eram pulados. Isto caracteriza esta amostra como do tipo sistemática.

Foram coletadas informações sobre características demográficas (idade materna e estado civil, cor da pele; idade e sexo da criança), socioeconômicas (escolaridade materna e renda familiar), condições de moradia (tipo de construção, tipo de piso, número de compartimentos usados para dormir, acesso à rede de esgotos e água tratada e disponibilidade de sanitário), assistência à gestação e ao parto (número de consultas de pré-natal realizadas, mês de inicio do pré-natal, doses de vacina antitetânica recebida, tipo de parto e de profissional que o realizou e peso ao nascer), acesso e utilização de serviços de saúde (ocorrência de hospitalizações nos 12 meses antecedentes à entrevista, realização de consultas médicas nos últimos três meses, cobertura vacinal básica e monitoração do crescimento), padrão de morbidade e consumo de medicamentos nos últimos 15 dias, amamentação e dieta e avaliação do estado nutricional.

Seis entrevistadoras foram selecionadas e treinadas para aplicação de questionários, todas com nível superior completo e experiência previa em inquéritos. Os questionários eram enviados para codificação das questões abertas, revisão final, dupla digitação e comparação. Todo esse processo foi realizado utilizando-se o programa Epi Info versão 6.04 .14

Utilizou-se teste do qui-quadrado com correção de Yates para as comparações entre proporções (tabelas $2 \times 2$ ), enquanto que na análise multivariada utilizou-se a regressão de Poisson com ajuste robusto da variância. A análise multivariada baseouse no modelo hierárquico previamente estabelecido. A significância estatística de cada variável no modelo foi avaliada através do teste de Wald. Em um primeiro momento, cada bloco de variáveis de um determinado nível foi incluído na análise, tendo sido mantidas no modelo final todas aquelas variáveis que mostraram associação $(p \leq 0,20)$ com o desfecho (administração de medicamento a crianças menores de cinco anos sem orientação médica).Estas análises foram realizadas no software Stata 11.2.15

Neste modelo, as variáveis situadas no mesmo nível e nos níveis anteriores foram consideradas como potenciais fatores de confusão na relação com o desfecho, enquanto as variáveis situadas em níveis posteriores foram consideradas como possíveis mediadores da associação. As variáveis, sele- cionadas em um determinado nível, permaneceram no modelo sendo consideradas como fatores de risco ou proteção para o desfecho em questão, mesmo que, com a inclusão de variáveis de níveis anteriores tivessem perdido sua significância estatística. ${ }^{16} \mathrm{O}$ controle de qualidade deste estudo foi feito através da repetição de cerca de $10 \%$ das entrevistas para perguntas-chave.

\section{Resultados}

No município de Caracol, foram incluídas 590 crianças menores de cinco anos. Destas, segundo as mães, $46 \%$ estiveram doentes nas duas semanas que antecederam à entrevista. A Tabela 1 mostra que para $30 \%$ delas foi dado algum medicamento sem orientação médica. As maiores prevalências de automedicação ocorreram entre mães de cor da pele preta (37\%), com 30 anos ou mais de idade (40\%), que viviam sem companheiro (36\%), que não completaram um único ano de escolaridade (41\%), que trabalharam fora de casa nos últimos 12 meses (31\%), que residiam entre 1 e $2,9 \mathrm{~km}$ dos serviços de saúde mais próximo (35\%), cujos filhos possuíam menos de um ano de idade (55\%), que haviam sido hospitalizados nos últimos 12 meses (39\%), e que procuraram o serviço de saúde, mas não conseguiram atendimento $(83 \%)$.

Esta mesma Tabela 1 mostra que no município de Caracol, mesmo após ajuste para diversos fatores de confusão conforme modelo previamente estabelecido, as seguintes variáveis mostraram-se significativamente associadas ao desfecho: idade materna, distância ao serviço de saúde e busca por consulta médica nos últimos 15 dias.

Em Garrafão do Norte, o estudo incluiu 1081 crianças menores de cinco anos. Segundo as mães, $41 \%$ delas estiveram doentes nos últimos 15 dias. Destas, $25 \%$ foram medicadas sem orientação médica. A Tabela 2 mostra que as maiores prevalências de automedicação ocorreram entre filhos cujas mães eram de cor da pele parda (26\%), tinham entre 25 e 29 anos de idade (29\%), possuíam nove anos ou mais de escolaridade $(32 \%)$, pertenciam ao tercil intermediário de renda (29\%), que haviam trabalhado nos 12 meses antecedentes ao parto, que residiam entre 1 e $2,9 \mathrm{~km}$ de algum tipo de serviço de saúde, que tiveram filhos hospitalizados duas vezes ou mais nos últimos 12 meses e que procuraram os serviços de saúde com o filho doente, mas não conseguiram atendimento (40\%).

A Tabela 2 mostra ainda que em Garrafão do Norte, mesmo após ajuste para fatores de confusão, mantiveram-se significativamente associadas à 
Tabela 1

Análise bruta e ajustada para automedicação em crianças menores de cinco anos. Caracol, PI, 2005.

\begin{tabular}{|c|c|c|c|c|c|c|}
\hline Variáveis & $\mathrm{N}$ & $\%$ & Bruta & $p$ & Ajustada & $p$ \\
\hline Cor da pele & & & & $0,04 \#$ & & $0,07 \# a$ \\
\hline Branca & 47 & 23,4 & 1,00 & & 1,00 & \\
\hline Parda/mulata & 416 & 28,6 & $1,22(0,71-2,10)$ & & $1,18(0,70-1,99)$ & \\
\hline Idade materna (anos) & & & & $0,04 \#$ & & $0,04 \# a$ \\
\hline $13-19$ & 198 & 24,8 & 1,00 & & 1,00 & \\
\hline $20-24$ & 226 & 32,3 & $1,31(0,96-1,78)$ & & $1,29(0,95-1,75)$ & \\
\hline $25-29$ & 96 & 28,1 & $1,14(0,76-1,70)$ & & $1,16(0,78-1,74)$ & \\
\hline$\geq 30$ & 70 & 40,0 & $1,62(1,11-2,35)$ & & $1,55(1,06-2,25)$ & \\
\hline $12-23,9$ & 118 & 26,3 & $1,44(0,80-2,62)$ & & $1,41(0,77-2,59)$ & \\
\hline $24-35,9$ & 117 & 33,3 & $1,83(1,03-3,25)$ & & $1,74(0,97-3,11)$ & \\
\hline$\geq 36$ & 246 & 32,1 & $1,77(1,03-3,04)$ & & $1,73(0,99-3,02)$ & \\
\hline Sexo da criança & & & & 0,40 * & & 0,40 * \\
\hline Masculino & 297 & 31,7 & 1,00 & & 1,00 & \\
\hline Feminino & 293 & 28,3 & $0,90(0,70-1,15)$ & & $0,90(0,70-1,14)$ & \\
\hline Estado civil & & & & $0,30 *$ & & $0,30 *$ \\
\hline Com companheiro & 543 & 29,5 & 1,00 & & 1,00 & \\
\hline Sem companheiro & 47 & 36,2 & $1,23(0,82-1,83)$ & & $1,23(0,83-1,81)$ & \\
\hline Tercil de menor renda & 213 & 30,5 & $0,98(0,73-1,32)$ & & $0,96(0,70-1,32)$ & \\
\hline Tercil intermediário de renda & 184 & 28,8 & $0,93(0,68-1,27)$ & & $0,91(0,70-1,27)$ & \\
\hline Tercil de maior renda & 190 & 31,1 & 1,00 & & 1,00 & \\
\hline Trabalho materno (últimos 12 meses) & & & & $0,50 *$ & & $0,80 *$ \\
\hline Não & 250 & 28,4 & 1,00 & & 1,00 & \\
\hline Sim & 340 & 31,2 & $1,10(0,85-1,41)$ & & $0,97(0,75-1,26)$ & \\
\hline Distância do posto de saúde $(\mathrm{km})$ & & & & 0,06 * & & $0,04 \# a$ \\
\hline$<1$ & 229 & 24,5 & 1,00 & & 1,00 & \\
\hline $1-2,9$ & 183 & 35,0 & $1,43(1,06-1,93)$ & & $1,51(1,11-2,04)$ & \\
\hline $3-5$ & 178 & 32,0 & $1,31(0,96-1,79)$ & & $1,37(1,00-1,88)$ & \\
\hline Déficit altura/idade ${ }^{\dagger}$ & & & & 0,90 * & & $0,70 *$ \\
\hline Não & 510 & 30,0 & 1,00 & & 1,00 & \\
\hline $\operatorname{Sim}$ & 79 & 30,4 & $1,01(0,71-1,45)$ & & $0,93(0,64-1,36)$ & \\
\hline Hospitalizações últimos 12 meses & & & & $0,30 *$ & & $0,10^{*}$ \\
\hline Nenhuma & 481 & 29,7 & 1,00 & & 1,00 & \\
\hline 1 & 71 & 26,8 & $0,90(0,60-1,35)$ & & $0,87(0,57-1,32)$ & \\
\hline$\geq 2$ & 38 & 39,5 & $1,33(0,87-2,02)$ & & $1,44(0,98-2,11)$ & \\
\hline
\end{tabular}


Análise bruta e ajustada para automedicação em crianças menores de cinco anos. Caracol, PI, 2005.

\begin{tabular}{|c|c|c|c|c|c|c|c|}
\hline Nível & Variáveis & $\mathrm{N}$ & $\%$ & Bruta & $p$ & Ajustada & $p$ \\
\hline \multirow[t]{5}{*}{4} & Consulta nos últimos 15 dias & & & & $<0,001$ * & & $<0,001 * a$ \\
\hline & Não procurou & 467 & 27,2 & 1,00 & & 1,00 & \\
\hline & Sim, pronto socorro & 29 & 20,7 & $0,76(0,37-1,58)$ & & $0,65(0,33-1,31)$ & \\
\hline & Sim, outro local & 9 & 33,3 & $1,23(0,48-3,13)$ & & $1,07(0,41-2,79)$ & \\
\hline & Total & 590 & 30,0 & & & & \\
\hline
\end{tabular}

† Escore z altura/idade <-2,0 conforme curvas da OMS; * Teste de Wald de heterogeneidade; \# Teste de Wald de tendência a Variáveis conservadas no modelo quando $p \leq 0,20$ na análise ajustada; b Ajustado para outras variáveis do nível 1 com $p \leq 0,20$, com exceção do índice de bens.

Tabela 2

Análise bruta e ajustada para automedicação em crianças menores de cinco anos. Garrafão do Norte, PA, 2005.

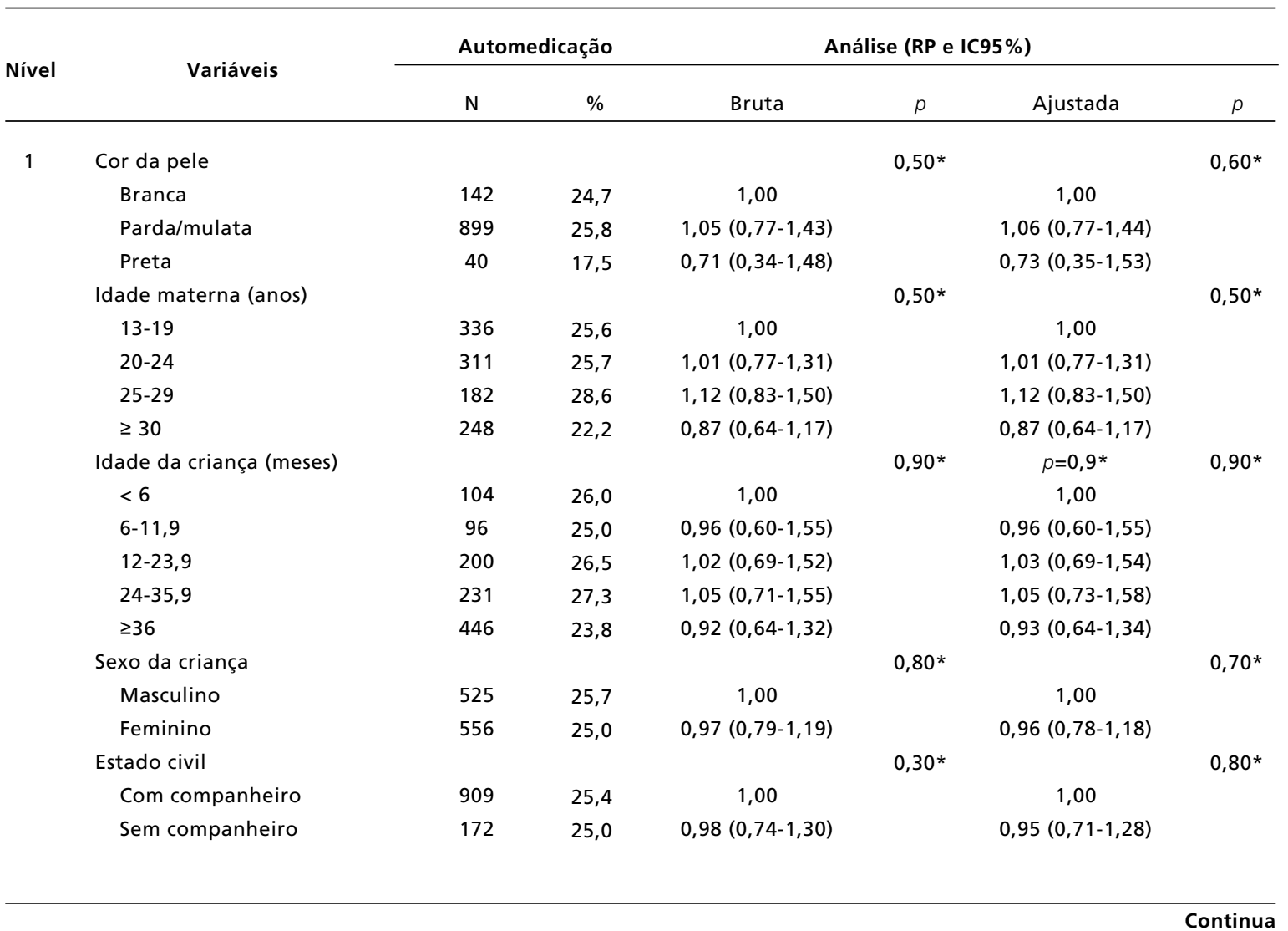


Análise bruta e ajustada para automedicação em crianças menores de cinco anos. Garrafão do Norte, PA, 2005.

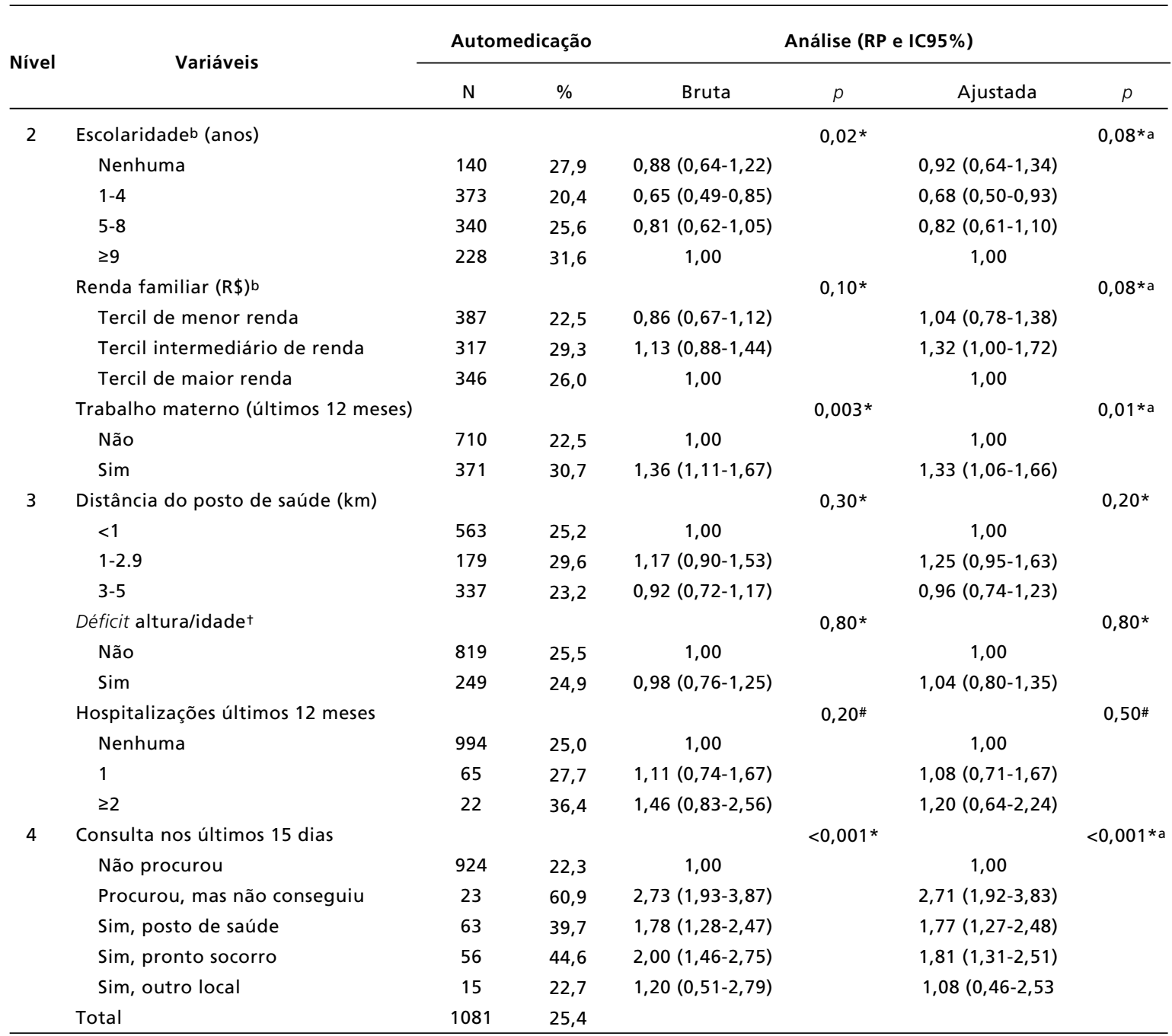

* Teste de Wald de heterogeneidade; \# Teste de Wald de tendência; a Variáveis conservadas no modelo quando $p \leq 0,20$ na análise ajustada; b Ajustado para outras variáveis do nível 1 com $p \leq 0,20$, com exceção do índice de bens.

automedicação as variáveis trabalho materno nos 12 meses anteriores ao parto e busca por consulta médica nos últimos 15 dias.

\section{Discussão}

Este estudo mostrou que a prevalência de automedicação entre menores de cinco anos nos dois municípios variou conforme algumas características das crianças, de suas mães e de suas famílias. Em Caracol, a análise ajustada mostrou que os fatores associados de forma independente à automedicação foram idade materna, distância da residência aos serviços de saúde e, sobretudo, a impossibilidade de realização de consulta médica nos últimos 15 dias.
Em Garrafão do Norte, as variáveis com efeito independente sobre o desfecho foram trabalho materno nos últimos 12 meses e, também, não conseguir atendimento médico para o filho nos últimos 15 dias.

Ao interpretar estes dados há que considerar que se trata de um estudo transversal, e que qualquer inferência sobre causalidade fica prejudicada. No entanto, isto não interfere nas estimativas de prevalência nem nos valores das medidas de efeito obtidas.

A prevalência de automedicação entre menores de cinco anos nos dois municípios foi inferior à observada em outros estudos.2,9,10-12,17 Em Caracol foi de $30 \%$, enquanto em Garrafão do Norte foi de $25 \%$. Esta diferença pode ser atribuída à diferença 
nas faixas etárias estudadas. A prevalência de automedicação entre crianças das diferentes coortes de estudo conduzidas em Pelotas mostrou importante variação entre 1982 e 2004. A prevalência de automedicação para crianças com idade entre $35 \mathrm{e}$ 53 meses de idade pertencentes à coorte de 1982 foi de $38 \%$; $^{2}$ na coorte de 1993 , para crianças com idade entre três e seis meses, a prevalência foi de $33 \%, 17$ enquanto que na coorte de 2004, aos 24 meses de idade, a prevalência chegou a 34\%.$^{9}$ Em Piracicaba e Limeira, que, além de crianças incluiu também adolescentes, a prevalência de automedicação foi de $57 \%, 10$ enquanto em Teresópolis e Tubarão foi de $50 \%$ e $74 \%$, respectivamente. 11,12 Em todos estes estudos, o período recordatório foi de 15 dias anteriores à entrevista. A principal dificuldade desta comparação reside na inexistência de artigo semelhante quanto à faixa etária estudada. No entanto, era de se esperar que a prevalência de automedicação nos dois municípios estudados fosse maior que a de outras localidades pela baixa disponibilidade de serviços de saúde e, sobretudo, pelo difícil acesso. Isto poderia favorecer a oferta de medicamentos à criança sem orientação médica. No entanto, há que destacar também a baixa disponibilidade de medicamentos no setor público em Caracol e Garrafão e, sobretudo, em decorrência da pobreza, a falta de recursos financeiros para adquiri-los junto ao setor privado.

Embora estas localidades sejam pobres, há importantes diferenças entre eles. Caracol, elevado á categoria de município em meados do século passado, possui infraestrutura em educação e saúde superior a de Garrafão do Norte. Sua população convive de forma crônica com a pobreza. O Programa Bolsa-Família do governo federal constituiu-se numa das mais importantes fontes de renda do município, visto atender cerca de dois terços das famílias. Garrafão do Norte foi emancipado em 1988. Sua infraestrutura em saúde e educação é bastante precária, sua extensão territorial cerca de quatro vezes maior do que a de Caracol Praticamente metade das famílias são cobertas pelo Bolsa-Família (www.beneficiossociais.caixa.gov.br/ consulta/beneficio/ 04.01.00-00 00.asp - acessado em 12/04/2012). Em ambos os municípios, a variável que mais fortemente mostrou-se associada ao desfecho foi à impossibilidade de o filho receber atendimento médico nos últimos 15 dias. Isto sugere que a dificuldade em obter atendimento nos serviços de saúde facilitou a automedicação destas crianças Este achado é diferente de outras localidades mais desenvolvidas, em que, apesar da maior oferta de serviços de saúde, a ocorrência de automedicação é também elevada. Neste caso, além de razões culturais, a facilidade na aquisição de medicamentos, a repetição de uma receita e a falta de medicamentos nos serviços de saúde podem contribuir para uma maior taxa de automedicação. $2,9,18-20$

A automedicação é mais comumente observada entre mães que mantém medicamentos estocados no domicílio, que se utilizam de receitas prévias fornecidas por médicos e que obtiveram previamente bom resultado com a mesma criança.7,11,21 Isto é mais facilmente observado no caso de doenças de longa duração como, por exemplo, asma, mas pode, também, ocorrer em casos de doenças comuns, particularmente febre, cólicas e resfriado comum.1012,20,21 No Brasil, há poucos estudos sobre o tema. Mais raro ainda é encontrar artigos discutindo políticas públicas com o objetivo de promover intervenções e estratégias de prevenção à automedicação. 22

Em vista dos efeitos nocivos da automedicação sobre a saúde infantil como, por exemplo, indução de resistência bacteriana, mascaramento de doenças e intoxicação medicamentosa, diversas tentativas têm sido feitas visando coibir esta prática bem como promover o uso racional de medicamentos, mas sem grande sucesso. Há, na verdade, indícios de que esta prática vem aumentando. Dados oriundos também das coortes de Pelotas, que incluíram todos os recém-nascidos da área urbana, revelam que a automedicação vem aumentando com o avançar da idade da criança, pelo menos entre menores de dois anos. A prevalência de automedicação passou de $11 \%$ aos três meses para $34 \%$ aos 24 meses. 9 Entre 35 e 53 meses, a taxa de automedicação chegou a $62 \% .^{2}$ A causa mais comum foi o reaproveitamento de prescrição médica: quanto mais prescrição, maior a ocorrência de automedicação. ${ }^{9}$ Em Limeira e Piracicaba, SP, metade das automedicações ocorreu por deliberação da mãe. ${ }^{10} \mathrm{E}$ o que facilita esta prática, além da disponibilidade de prescrição anterior, é o armazenamento de medicamentos no próprio domicilio e facilidade de aquisição nas farmácias. 10,11,19,21

Os dados aqui apresentados mostram a necessidade de, nestes municípios, aumentar a disponibilidade de serviços médicos, facilitar o acesso geográfico aos serviços de saúde e realizar campanha mostrando os riscos da automedicação. Espera-se que estas iniciativas reduzam a ocorrência de intoxicação medicamentosa, prática comum e crescente entre menores de cinco anos nas mais diferentes regiões do país, como vem sendo denunciado pelo Sistema Nacional de Informações TóxicoFarmacológicas. Intervenções coletivas visando 
desencorajar esta prática vêm sendo apontadas como uma ação prioritária em saúde infantil.22

\section{Referências}

1. Organización Mundial de La Salud. Promoción del uso racional de medicamentos: componentes centrales. Ginebra; 2002. (Perspectivas políticas sobre medicamentos de la OMS, 5).

2. Béria JU, Victora CG, Barros FC, Teixeira AB, Lombardi C. Epidemiologia do consumo de medicamentos em crianças de centro urbano da região sul do Brasil. Rev Saúde Pública. 1993; 27: 95-104.

3. Barros JAC. Nuevas tendências de La medicalización. Ciênc Saúde Coletiva. 2008; 13 (Suppl.): 579-87.

4. Fundação Oswaldo Cruz/Centro de Informação Científica e Tecnológica/Sistema Nacional de Informações TóxicoFarmacológicas. Estatística Anual de Casos de Intoxicação e Envenenamento. Brasil, 2008. [Acesso em 14 ago 2010]. Disponível em: http://www.fiocruz.br/sinitox

5. ANVISA (Agência Nacional de Vigilância Sanitária). Portaria $\mathrm{n}^{\circ} 3.916$, de 30 de Outubro de 1998. Aprova a Política Nacional de Medicamentos. [Acesso em 18 out 2008]. Disponível em: http://www.anvisa.gov.br.

6. Zanine AC, Paulo LG. Automedicação no Brasil. Rev Assoc Med Bras. 1988; 34: 69-75.

7. Vilarino JF, Soares IC, Silveira CM, Rödel PP, Bortoli R, Lemos RR. Perfil da automedicação em município do Sul do Brasil. Rev Saúde Pública. 1998; 32: 43-9.

8. Arrais OS, Coelho HI, Batista MC, Righi RE, Arnau JM. Perfil da automedicação no Brasil. Rev Saúde Pública. 1997; 31: 71-7.

9. Oliveira EA, Bertoldi AD, Domingues MR, Santos IS, Barros AJD. Uso de medicamentos do nascimento aos dois anos: coorte de nascimentos de Pelotas, RS, 2004. Rev Saúde Pública. 2010; 44: 591-600.

10. Pereira FSVT, Bucaretchi F, Stephan C, Cordeiro R Automedicação em crianças e adolescentes. J Pediatr. 2007; 83: 453-58.

11. Ribeiro JM, Siqueira SAV, Pinto LFS. Avaliação da atenção à saúde da criança (0-5 anos) no PSF de Teresópolis (RJ) segundo a percepção dos usuários. Ciênc Saúde Coletiva. 2010; 15: 517-27.

12. Cancelier ACL, Kubo TK, Pizzol FD. Automedicação em crianças com rinofaringite aguda. Arq Catarin Med. 2006; 35: $35-40$.

Recebido em 17 de fevereiro de 2012

Versão final apresentada em 16 de março de 2012

Aprovado em 9 de abril de 2012
13. IBGE (Instituto Brasileiro de Geografia e Estatística) 2009. Cidades. [Acesso em 23 jun 2010]. Disponível: http://www.ibge.gov.br/cidadesat/topwindow.htm?1

14. Dean AG, Dean JA, Coulombier D, Brendel KA, Smith DC, Burton AH, Dicker RC, Sulliven K, Fagan RF, Arner TG. Epi Info, Version 6: A word processing, database, and statistics program for epidemiology on microcomputers. Atlanta: Centers of Disease Control and Prevention; 1994.

15. StataCorp. Stata statistical software: release 7.0. CollegeStation: Stata Corporation; 2001.

16. Victora CG, Huttly SR, Fuchs SC, Olinto MT. The role of conceptual frameworks in epidemiological analysis: a hierarchical approach. Int J Epidemiol. 1997; 26: 224-7.

17. Weiderpass E, Béria JU, Barros FC, Victora CG, Tomasi ET, Halpern R. Epidemiologia do consumo de medicamentos no primeiro trimestre de vida em centro urbano do sul do Brasil. Rev Saúde Pública. 1998; 32: 335-44.

18. Winkelstein ML, Huss K, Butz A, Eggleston P, Vargas P, Rand C. Factors associated with medication self-administration in children with asthma. Clin Pediatric. 2000; 39: $337-45$.

19. Okumura J, WakaiI S, Umenai T. Drug utilisation and selfmedication in rural communities in Vietnam. Soc Sci Med. 2002; 54: 1875-86.

20. Halder S, Thaver IH. Self medication or self care: implication for primary health care strategies. J. Pak Med Assoc. 1995; 45: 297-8.

21. Mastroianni PC, Lucchetta RC, Sarra JR, Galduróz JCF. Estoque doméstico e uso de medicamentos em uma população cadastrada na estratégia saúde da família no Brasil. Rev Panam Salud Publica. 2011; 29: 358-64.

22. Pfaffenbach GMA, Tourinho FS, Bucaretchi F. Selfmedication among children and adolescents. Curr Drug Saf. 2010; 5 : 324-8. 